\title{
Material of Choice in Pediatric Cranioplasty
}

\author{
Anooja Abdul Salam ${ }^{1, \odot ~ I m o g e n ~}$ Ibbett $^{2} \quad$ Nova Thani' \\ ${ }^{1}$ Department of Neurosurgery, Royal Hobart Hospital, Affiliated to \\ University of Tasmania, Hobart, Tasmania, Australia \\ ${ }^{2}$ Department of Spine, Mater Hospital, Brisbane, Australia
}

\begin{abstract}
Address for correspondence Anooja Abdul Salam, MBBS, MPH, Department of Neurosurgery, Royal Hobart Hospital, Affiliated to University of Tasmania, 48 Liverpool Street, Hobart, Tasmania 7000, Australia (e-mail: abcanu@gmail.com).
\end{abstract}

Indian J Neurosurg 2021;10:103-107.

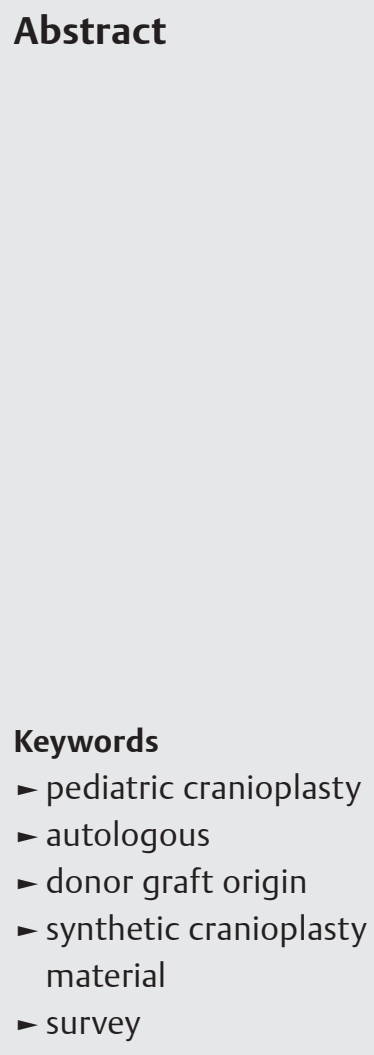

Background Current evidence is lacking regarding the optimum material required for cranioplasty in the pediatric population when native bone cannot be replaced. The aim of our survey was to examine current practice in Australia and New Zealand regarding pediatric cranioplasty material.

Methods The online tool SurveyMonkey was used to survey 244 neurosurgeons in Australasia. The survey consisted of five questions concerning preference of material and donor origin for pediatric cranioplasty.

Results Twenty-two neurosurgeons (9\%) participated. The results indicate that with small skull defects $(<3 \mathrm{~cm})$ in patients aged 0 to 2years, conservative management with observation alone is the preferred option (65\%). In patients aged 3 to 10 years, autologous donor bone was the most popular option, whereas for 11+ years, hydroxyapatite (HA) was the material of choice, followed by titanium. For defects of more than $3 \mathrm{~cm}$, autologous donor bone was preferred in under 11 years. In patients older than 11 years, titanium was the preferred choice $(46.67 \%)$. The preferred donor origin for autologous cranioplasty in small skull defects $(<3 \mathrm{~cm})$ was split calvarial grafts for all age groups. However, $68.42 \%$ of respondents managed those under 2 years conservatively. In large skull defects $(>3 \mathrm{~cm})$, the preferred donor origin was split calvarial grafts for patients older than 3 years (48.3\%). In patients aged 0 to 2 years, exchange cranioplasty was the preferred option when cranioplasty was performed.

Conclusion The current practice in Australia and New Zealand is to use autologous donor bone in preference to synthetic materials for cranioplasty in children under 11 years. In children older than 11 years, hydroxyapatite and titanium are the materials of choice.

\section{Introduction}

A literature review was previously conducted to investigate the materials used in pediatric cranioplasty. ${ }^{1}$

It was concluded that particulate bone grafts or exchange cranioplasty were commonly used in infants. ${ }^{1}$ In older children, custom-made implants using titanium or hydroxyapatite were more frequently used. ${ }^{1}$ However, the conclusion from the review was that there was not enough evidence to recommend an ideal pediatric cranioplasty material for all

published online

April 19, 2021
DOI https://doi.org/

10.1055/s-0040-1716933 ISSN 2277-954X. age groups. ${ }^{1}$ All the existing studies have very small sample sizes, which makes it difficult to develop an age-based protocol for pediatric patients. Hence, we conducted a survey to explore the current practice among the neurosurgeons in Australia and New Zealand.

The research questions in this study were aimed at examining the material of choice for pediatric cranioplasty in four different age groups of children: 0 to 2, 3 to 5,6 to 10 , and $11+$ years of age. The survey also explored the preferred donor site for autologous cranioplasty.
(C) 2021. Neurological Surgeons' Society of India.

This is an open access article published by Thieme under the terms of the Creative Commons Attribution-NonDerivative-NonCommercial-License, permitting copying and reproduction so long as the original work is given appropriate credit. Contents may not be used for commercial purposes, or adapted, remixed, transformed or built upon. (https://creativecommons.org/licenses/by-nc-nd/4.0/).

Thieme Medical and Scientific Publishers Pvt. Ltd. A-12, 2nd Floor, Sector 2, Noida-201301 UP, India 


\section{Methods}

The survey was conducted with the online survey tool Survey Monkey. The survey was sent via email to 244 consultant members of the Neurosurgical Society of Australasia.

The survey explored preferences in different materials used for pediatric cranioplasty surgeries as well as preferred donor site for autologous cranioplasty, based on patient age and size of defect. The data were analyzed using the tool "Survey Monkey" and the results are discussed below.

The survey consisted of the following questions:

1. How many pediatric neurosurgical operations do you perform per year?

2. When native bone cannot be replaced, what is your preferred material for cranioplasty in small skull defects $(<3 \mathrm{~cm})$ ?

3. When native bone cannot be replaced, what is your preferred material for cranioplasty in large skull defects $(>3 \mathrm{~cm})$ ?

4. If using autologous cranioplasty for small defects $(<3 \mathrm{~cm})$, what is your preferred donor origin?

5. If using autologous cranioplasty for large defects $(>3 \mathrm{~cm})$, what is your preferred donor origin?

\section{Results}

Responses were received from 22 neurosurgeons (9\%) out of 244 contacted.

\section{Question 1}

How many pediatric neurosurgical operations do you perform every year?

The survey results showed that approximately $32 \%$ of the respondents perform more than 50 pediatric neurosurgical cases annually, as shown in - Table 1. However,

Table 1 Percentage of surgeons categorized by the number of surgeries performed annually

\begin{tabular}{|l|l|}
\hline $\begin{array}{l}\text { Number of operations } \\
\text { performed annually }\end{array}$ & $\begin{array}{l}\text { Surgeons \%, } \\
\text { (number of surgeons) }\end{array}$ \\
\hline$<10$ & $50 \%(11)$ \\
\hline $11-30$ & $13.64 \%(3)$ \\
\hline $31-50$ & $4.55 \%(1)$ \\
\hline$>50$ & $31.82 \%(7)$ \\
\hline
\end{tabular}

approximately $50 \%$ of the neurosurgeons perform less than 10 pediatric neurosurgeries annually.

\section{Question 2}

When native bone cannot be used, what is your preferred material for cranioplasty in SMALL skull defects $<3 \mathrm{~cm}$ ?

A total of 20 respondents answered this question (two respondents chose not to answer).

The percentages of surgeons who opted for each type of cranioplasty (titanium, hydroxyapatite [HA], methylmethacrylate [MMA], autologous and none), in treating a small skull defect of $<3 \mathrm{~cm}$ in different pediatric age groups are given in -Table 2 and - Fig. 1 .

The results indicate that with small skull defects $(<3 \mathrm{~cm})$ in patient's aged 0 to 2 years, conservative management with observation alone is the preferred option (65\%), as shown in - Table 2. In patients aged 3 to 5 years and 6 to 10 years, autologous donor was the most popular option (35\% and $40 \%$, respectively), whereas for $11+$ years, HA was the material of choice (38.89\%), followed by titanium (27.78\%) ( - Fig. 1).

\section{Question 3}

When native bone cannot be used, what is your preferred material for cranioplasty in large skull defects $>3 \mathrm{~cm}$ ?

A total of 20 respondents answered this question. The percentages of surgeons who opted for each material (titanium, hydroxyapatite $[\mathrm{HA}]$, methylmethacrylate [MMA], autologous and none) in treating large skull defects of $>3 \mathrm{~cm}$ in different pediatric age groups are given in $\boldsymbol{\sim}$ Table $\mathbf{3}$ and $\boldsymbol{\sim}$ Fig. $\mathbf{2}$.

In cases with a defect more than $3 \mathrm{~cm}$, autologous donor was the preferred option in patients aged 3 to 10 years. In

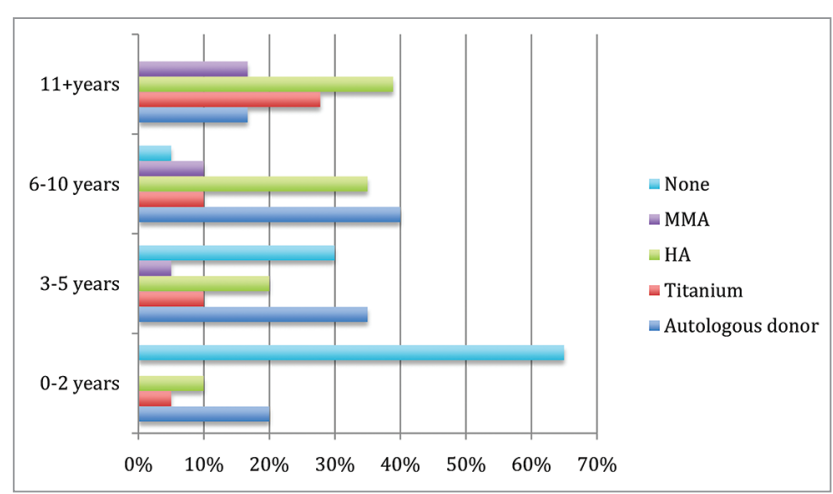

Fig. 1 Percentage of surgeons who prefer each cranioplasty material in skull defects $<3 \mathrm{~cm}$ across different pediatric age groups.

Table 2 Percentage of surgeons who prefer each cranioplasty material in skull defects $<3$ cm across different pediatric age groups

\begin{tabular}{|c|c|c|c|c|c|}
\hline Age groups (years) & Autologous donor (\%) & Titanium (\%) & $\mathrm{HA}(\%)^{\mathrm{a}}$ & MMA $(\%)^{\mathrm{b}}$ & None (\%) \\
\hline $0-2$ & 20 & 5 & 10 & 0 & 65 \\
\hline $3-5$ & 35 & 10 & 20 & 5 & 30 \\
\hline $6-10$ & 40 & 10 & 35 & 10 & 5 \\
\hline $11+$ & 16.67 & 27.78 & 38.89 & 16.67 & 0 \\
\hline
\end{tabular}


Table 3 Percentage of surgeons who prefer each cranioplasty material across different age groups with respect to skull defects of $(>3 \mathrm{~cm})$

\begin{tabular}{|l|l|l|l|l|l|}
\hline Age groups (years) & Autologous donor (\%) & Titanium (\%) & HA (\%) & MMA (\%) & None (\%) $^{\text {b }}$ \\
\hline $0-2$ & 36.84 & 10.53 & 15.79 & 0 & 36.84 \\
\hline $3-5$ & 47.37 & 21.05 & 26.32 & 0 & 5.26 \\
\hline $6-10$ & 31.58 & 26.32 & 31.58 & 10.53 & 0 \\
\hline $11+$ & 6.67 & 46.67 & 20 & 26.67 & 0 \\
\hline
\end{tabular}

aHydroxyapatite.

'Methymethacrylate.

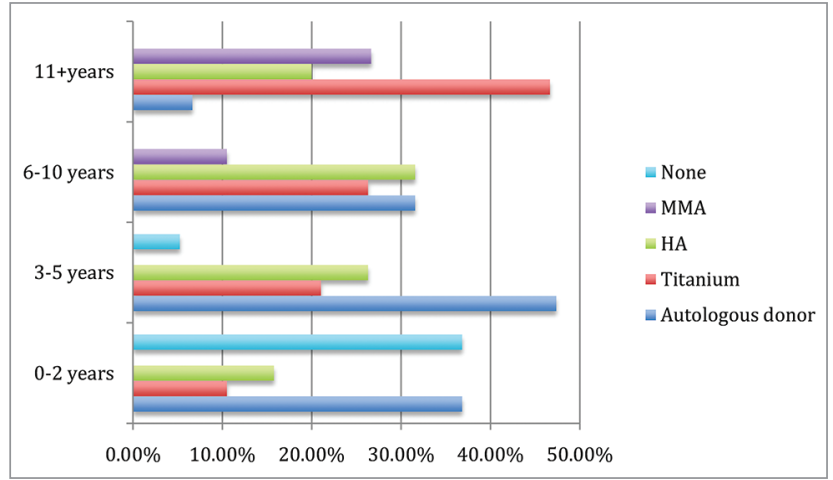

Fig. 2 Percentage of surgeons who prefer each cranioplasty material in skull defects $>3 \mathrm{~cm}$ across different pediatric age groups.

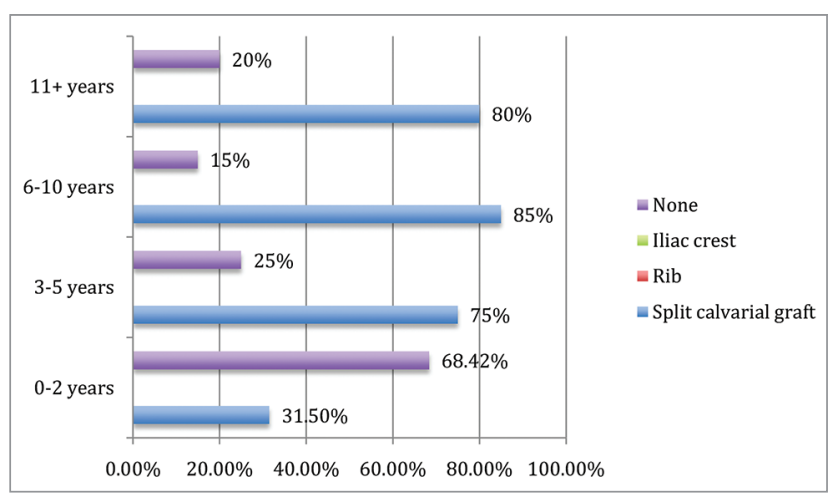

Fig. 3 Percentage of surgeons who opted for each donor origin across different age groups for $<3 \mathrm{~cm}$ skull defects.

patients above 11 years of age, titanium was the preferred choice in $46.67 \%$ of respondents (- Table 3 ). In the age group 0 to 2 years, the majority of respondents preferred a conservative approach $(37 \%)$ or autologous cranioplasty (37\%) (-Table 3).

MMA was commonly preferred for patients above 6 years of age. About $10 \%$ of respondents chose MMA in those between 6 to 10 year of age and approximately 27\% chose MMA for $11+$ years (-Fig. 2 ).

\section{Question 4}

If using autologous cranioplasty for small skull defects $<3 \mathrm{~cm}$, what is your preferred donor origin?

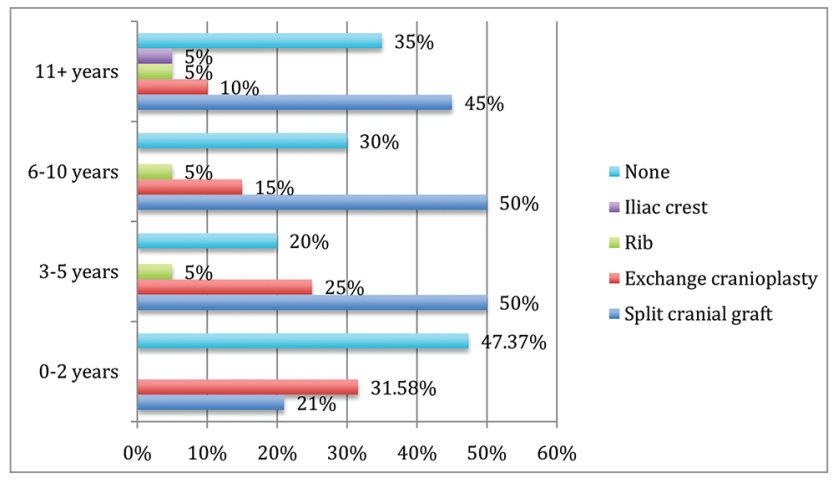

Fig. 4 Percentage of surgeons who opted for each donor origin across different age groups for $>3 \mathrm{~cm}$ skull defects.

A total of 20 respondents answered this question. The donor origin preferred by surgeons for $<3 \mathrm{~cm}$ skull defects in different age groups is shown in - Fig. 3.

When using autologous cranioplasty for small skull defects $(<3 \mathrm{~cm})$, the preferred donor origin was split calvarial grafts for all age groups, except in patients aged 0 to 2 years ( - Fig. 3). In patients aged 0 to 2 years, a conservative "watch and wait" approach was preferred by $68.42 \%$ of respondents, compared with split calvarial graft, which was preferred by $31.58 \%$ of respondents ( - Fig. $\mathbf{3}$ ).

\section{Question 5}

If using autologous cranioplasty for large defects $>3 \mathrm{~cm}$, what is your preferred donor origin?

A total of 20 respondents answered this question. The donor origin preferred by surgeons in different age groups for $>3 \mathrm{~cm}$ skull defects is shown in - Fig. 4 .

For autologous cranioplasty in large skull defects $(>3 \mathrm{~cm})$, the preferred donor origin was split calvarial graft for ages 3 to 5 years, 6 to 10 years and $11+$ years (50\%, 50\% and 45\%, respectively). In patients aged 0 to 2 years, a conservative "watch and wait" approach was again the preferred option ( $47.37 \%$ of respondents), followed by exchange cranioplasty (31.58\%) as seen in - Fig. 4.

Other materials have also been reported by the respondents for treatment of $>3 \mathrm{~cm}$ defects in children of more than 2 years of age. These include custom acrylic cranioplasty made using CT models and floating bone. Porous polyethylene was used by one neurosurgeon in all age groups and in all size defects. 


\section{Discussion}

The present study aimed to evaluate the current practice of neurosurgeons in Australia and New Zealand with regard to pediatric cranioplasty material. The reason for conducting this study was a lack of strong evidence as to the optimum material to use for cranioplasty in pediatric patients. All the existing studies have very small sample sizes, making it difficult to develop an age-based protocol for choosing optimal material.

The limitations of this study include the small cohort of respondents (22) with $9 \%$ response rate. However, this was the expected response, given the lower number of surgeons who specialize in pediatric neurosurgery. To ensure maximum response, multiple reminders were sent to all the participants. Another limitation is the low number $(<10)$ of pediatric cases performed by $50 \%$ of participating surgeons. Furthermore, this study is not a review of cases performed. It is indicative of the preference of individual surgeons, and may not be representative of what actually occurs in practice. Individual practice could vary based on the experience of the surgeon in using a particular material, availability and cost of the material. Other patient factors may also determine the specific management, such as the indication for non-native cranioplasty.

The cranial vault grows rapidly in the first 2 years and is stimulated by the growth of the brain, which reaches approximately $67 \%$ of its adult size within the first 2 years of life. ${ }^{2}$ The calvarium then continues to grow in a linear fashion to the brain, reaching adult size between 6 to 10 years of age. ${ }^{2}$ This pattern of growth determined the age groups used in our survey and served as a rationale in considering $11+$ age group as similar to adults.

The majority (65\%) of participating neurosurgeons preferred conservative management for defects less than $3 \mathrm{~cm}$ and did not offer cranioplasty in those under 2 years of age. Some of these respondents indicated that they monitor patients in this group for bone growth into the defect. This may mean that no cranioplasty is required or, if a small defect persists, autologous cranioplasty can be considered when the patient is older. This is consistent with evidence in the literature regarding bone formation in this age group. According to Opperman, new bone formation occurs at the sutural edges of the bone fronts in response to signals from growing neurocranium and continually undergo remodeling to accommodate, protect, and keep pace with the growing brain. ${ }^{2,3}$ Hence, conservative treatment is a safe and well-recognized management option for this young age group. Smaller skull defects have a greater chance of closing without any intervention.

Split calvarial grafts or exchange cranioplasty were the most preferred techniques for defects greater than $3 \mathrm{~cm}$ in all age groups up to 10 years. There are various studies supporting the use of split calvarial graft, particulate graft and exchange cranioplasty. ${ }^{1}$ They show good success rates and minimal complications for patients up to 22 years of age. ${ }^{1}$
In large skull defects of more than $3 \mathrm{~cm}$ in the $11+$ age group, titanium is the most commonly preferred material, followed by MMA and HA. The literature supports safe use of custom-made HA and titanium in children above 7 years of age. ${ }^{1}$ Stefani et al conducted a study of custom-made porous HA implants for cranioplasty in 114 pediatric patients aged 7 to 14 years. ${ }^{4}$ There was no report of early fracture or infection and only $5 \%$ late, posttraumatic fracture was reported. ${ }^{4}$

One of the respondents indicated a preference for the use of floating bone cranioplasty in $>3 \mathrm{~cm}$ defects in children of more than 2 years of age. The "floating" technique is thought to facilitate controlled volume expansion while reducing stretching and secondary damage to the edematous brain. ${ }^{5}$ As per Gutman et al, floating anchored craniotomy could be offered instead of decompressive craniectomy in certain cases of traumatic brain injury. However, there is only data available of this technique performed in 57 adults with an average age of 37.2 years. ${ }^{5}$ Further prospective trials are warranted to further assess the safety and utility of this procedure in the pediatric population. ${ }^{5}$

\section{Conclusion}

The survey results indicate that current practice in Australia and New Zealand for cranioplasty in relation to patient age and size of defect is consistent with the currently available best evidence, which was discussed in detail in the previously published literature review ${ }^{1}$

The preferred material for cranioplasty is autologous bone with split calvarial graft or exchange cranioplasty for defects less than $3 \mathrm{~cm}$ in in children aged 3 to 11 years. In children less than 2 years, a conservative approach is preferred. In children older than 11 years, HA and titanium are the materials of choice.

In autologous cranioplasty, the preferred donor origin for patients above 3 years of age is split calvarial graft, irrespective of the size of skull defect. For children under 2 years of age, when opting for surgical management, split calvarial graft for small defects and exchange cranioplasty for large skull defects were the preferred donor origins.

A larger study comparing different cranioplasty materials in the pediatric population with long-term follow up would be useful to ascertain safety and efficacy before developing a universally accepted protocol.

\section{Note}

The Tasmanian Health and Medical Human Research Ethics Committee have approved this study. The abstract was published in the AANSIM conference abstract book in December 2019.

\section{Funding \\ None.}

\section{Conflict of Interest}

None declared. 


\section{References}

1 Salam AA, Ibbett I, Thani N. Paediatric cranioplasty: a review. Interdiscip Neurosurg 2018;13:59-65

2 Flaherty K, Singh N, Richtsmeier JT. Understanding craniosynostosis as a growth disorder. Wiley Interdiscip Rev Dev Biol 2016;5(4):429-459

3 Opperman LA. Cranial sutures as intramembranous bone growth sites. Dev Dyn 2000;219(4):472-485
4 Stefini R, Esposito G, Zanotti B, Iaccarino C, Fontanella MM, Servadei F. Use of "custom made" porous hydroxyapatite implants for cranioplasty: postoperative analysis of complications in 1549 patients. Surg Neurol Int 2013;4:12

5 Gutman MJ, How E, Withers T. The floating anchored craniotomy. Surg Neurol Int 2017;8:130 